\title{
Effect of EVA lesion on reversal learning in the dark
}

\author{
A. B. CARRAN AND L. E. ANDERSON 1 \\ SUNY, COLLEGE AT BROCKPORT
}

Albino rats were pretrained in a water $T$ maze in the light. Experimental Ss were then given lesions in the extrinsic visual area of neocortex (EVA) and in surrounding neocortex. Control Ss were given lesions in neocortex surrounding EVA to equate groups for the amount of damage outside EVA. Ss were then trained in the dark for five reversals and it was found that experimental Ss learned more slowly than controls $(p<.005)$.

The work of Tsang (1936), and Orbach (1959) does not provide conclusive evidence that ablation of the extrinsic visual area $(E V A)^{2}$ causes deficit in learning and retaining the maze habit without visual cues since lesions made by these investigators extended outside EVA. Finley (1941) confined damage to EVA. Her lesioned and nonlesioned Ss performed equivalently but lesions averaged only $10 \%$ of the neocortex. Since a "critical mass" of lesion may be required to affect behavior in a given experiment, the experiment reported herein was designed to test for an effect of large lesions of EVA, using a control for damage unavoidably inflicted outside EVA.

\section{Subjects and Apparatus}

The Ss were 28 male albino rats, 68-86 days of age, and $270-360 \mathrm{~g}$ on the day of pretraining. The apparatus was a water $T$ maze measuring 15 in. inside depth and filled to a depth of 11 in. with water at room temperature. The stem of the maze was $10 \mathrm{in.}$ wide and 13 in. long. A start box at the base of the stem (7.5 in. long and 5 in. wide) was hinged to swing upward and was grooved inside at water level to hold a removable Plexiglas floor. The cross-bar of the maze was $10 \mathrm{in}$. wide and $54 \mathrm{in.} \mathrm{long.} \mathrm{The} \mathrm{maze} \mathrm{was} \mathrm{flat} \mathrm{black} \mathrm{except}$ for flat white stripes, $.75 \mathrm{in}$. wide and .75 in. apart, along the inside of the right alley above water level. The apparatus was housed in a darkroom.

\section{Procedure}

The Ss were paired. With the exception of Ss 19 and 20, both given control lesions, one member of each pair was assigned randomly to the EVA lesion group and the other to the control lesion group after the day of pretraining had been set. Pretraining was conducted in the light and consisted of 10 consecutive trials, the order of correct choice being RLRLLRRRLL. For each trial $E$, blind to the treatment of the $S$, placed $S$ in the start box and set a Lafayette Model 5432 timer modified to time $1 \mathrm{~min}$. When the timer finished ringing, $\mathrm{E}$ removed the floor of the start box and swiftly rotated it, releasing $S$, Then $E$ placed a hand at the end of each alley, fingers spread and extending into the water. If $S$ touched the hand in the incorrect alley (incorrect choice), $E$ withdrew the hand abruptly, leaving $S$ in the water until $\mathbf{S}$ touched the other hand. When $\mathbf{S}$ touched the hand in the correct alley, $E$ removed $S$ and scored the trial.

Five hours after pretraining $\mathrm{S}$ was lesioned. On the day following surgery $S$ rested. On the succeeding day $S$ was begun on reversal training, 10 trials daily. The procedure was identical to that of pretraining except that the light was off and, beginning with the left alley, the same alley remained correct throughout a session. The criterion for reversal was 9 out of 10 correct for a given session. Training was carried through five reversals.

\section{Lesions}

Surgery and Histology. The lesions were made by aspiration with $S$ under pentobarbital sodium anesthesia. After surgery, $\mathrm{S}$ was returned to its home cage where tetracycline hydrochloride was available. Four weeks after surgery $S$ was perfused intracardially, the brain removed and photographed, and the lesion sketched. Coronal sections at 50 micra intervals were made through the dorsal nucleus of the lateral geniculate (LGN) and stained with thionin. Sections were also taken at 50-200 micra, as desired, throughout areas of lesion and examined at $30 \mathrm{X}$ magnification.

The location of lesions and the results of histology

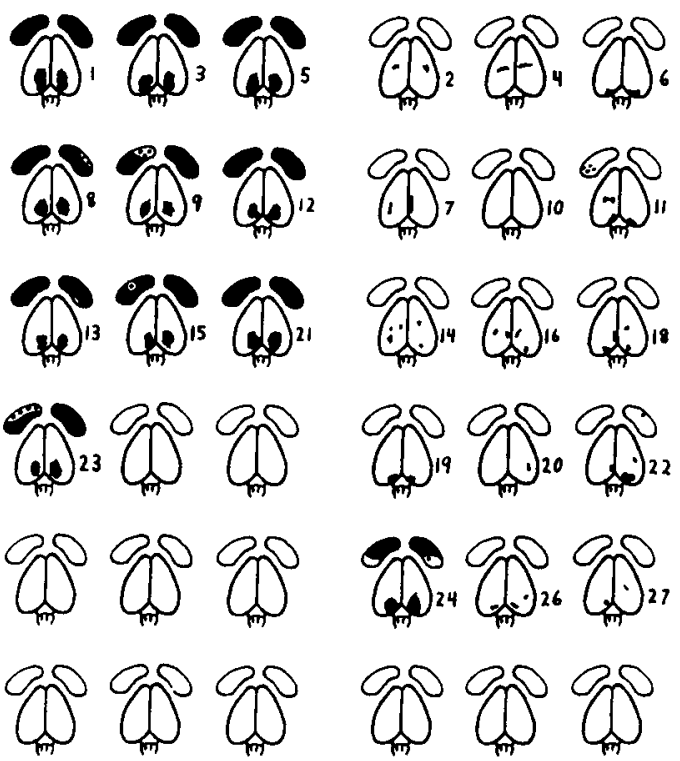

Fig. 1. Location of neocortical lesions and associated retrograde de generation of LGN for individual Ss of experimental and control groups. Black indicates severe and dots indicate moderate degeneration of LGN. Numbers refer to the order in which Ss began the experiment and indicate the pairing of Ss. 


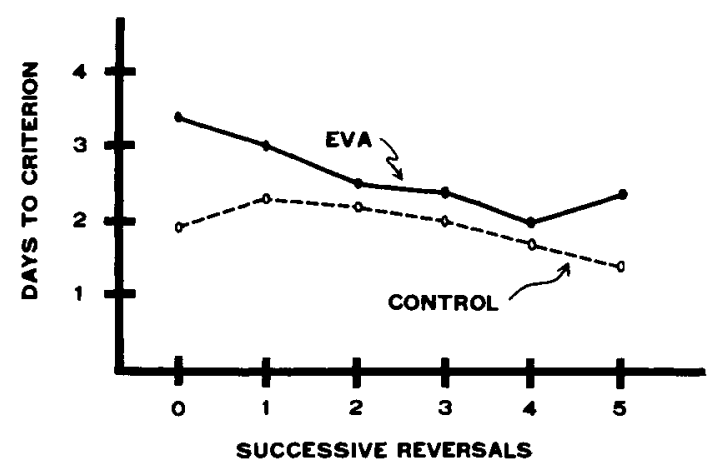

Fig. 2. The effect of EVA lesion on reversal learning.

are shown as Fig 1. Not shown in Fig 1 are the brain lesions of discarded Ss 17, 25, and 28. A cyst was discovered in the brain of S 28 and Ss 17 and 25 had superficial damage to the dorsal hippocampus. All neocortex was removed but no hippocampal damage was discovered in the remaining Ss of the EVA lesion group. Control group lesions were of good depth except one lesion only for each of Ss 4, 7, 24, 26 and 27. In these cases the lesion extended through at least one-half of the neocortex. Subject 24 incurred complete removal of neocortex of the posterior third in both lesions but some neocortex remained in the anterior third of the lesion on the left side.

\section{Resulis and Discussion}

Damage outside EVA totaled $5-20 \mathrm{~mm}^{2}$ greater in the remaining experimental Ss than in paired controls and it was decided to replace $S 10$, a fast learner without lesion, with $\mathrm{S} 19$ in order to achieve a fair degree of match between groups for damage outside EVA. The results of the groups so formed are shown as Fig 2. The EVA lesion group learned significantly more slowly than the control group $(F=13.2, \mathrm{df}=1 / 18, \mathrm{p}<.005)$. The main effect of reversal number did not attain significance $(F=1.87, d f=5 / 90, .25>p>.10)$ and the reversal number times lesion interaction did not approach significance.

The present results are consistent with Lashley's hypothesis of general mass action (an hypothesis which is unfortunately cast in null form) and with electrophysiological evidence (Jung, 1958) of vestibular as well as nonspecific thalamic input to visual cortex. Since aspiration was used in the present study, it is possible that the obtained result was due to undetected damage to subcortical fibers, but it is tentatively concluded that EVA functions in $T$ maze reversal training in the dark.

\section{References}

FINLEY, C. B. Equivalent losses in accuracy of response after central and after peripheral sense deprivation. J. comp. Neurol, 1941, 74, 203-237.

JUNG, R. Coordination of specific and nonspecific afferent impulses at single neurons of the visual cortex. In Jasper, H. H. (Ed.) Reticular formation of the brain. Boston: Little, Brown, 1958. Pp. 423-434.

LASHLEY, K. S. The mechanism of vision. VIII. The projection of the retina upon the cerebral cortex of the rat. J. comp. Neurol, 1934, $60,57-80$.

LASHLEY, K. S. Studies of cerebral function in learning. XII. Loss of the maze habit after occipital lesions in blind rats. J. comp. Neurol., $1943,79,431-462$.

PRIBRAM, K. H. Neocortical functions in behavior. In H. F. Harlow \& C. N. Woolsey (Eds.) Biological and biochemical bases of behavior. Madison: Univ. of Wisconsin Press, 1958. Pp. 151-172.

ROSE, J. E., \& WOOLSEY, C. N. Organization of mammalian thalamus and its relationship to the cerebral cortex. Electroencephalog. clin. Neurophysiol., 1949, 1, 391-404.

ROSE, J. E., \& WOOLSEY, C. N. Cortical connections and functional organization of the thalamic auditory system of the cat. In $\mathrm{H}$. F Harlow, \& C. N. Woolsey (Eds.) Biological and biochemical bases of behavior. Madison: University of Wisconsin Press, 1958. Pp. 127-150.

TSANG, Y. C. The function of the visual areas of the cerebral cortex of the rat in the learning and retention of the maze II. Comp. Psychol Monogr., 1936, 12, No. 57.

Notes

1. Now at the University of Rochester.

2. Included in EVA (Rose \& Woolsey; 1949, 1958) (Pribram, 1958) is that part of the striate cortex destruction of which causes retrograde degeneration of the lateral geniculate of the thalamus (Lashley, 1934). 